\title{
Matrix metalloproteinase 7 is required for tumor formation, but dispensable for invasion and fibrosis in SMAD4-deficient intestinal adenocarcinomas
}

\author{
Takanori Kitamura ${ }^{1,3}$, Kyoko Biyajima $^{1,3}$, Masahiro Aoki ${ }^{1}$, Masanobu Oshima ${ }^{2}$ and Makoto M Taketo ${ }^{1}$
}

Expression of matrix metalloproteinase 7 (MMP7) is increased in the human colorectal carcinomas, and correlates with malignant progression. However, its contribution to colon cancer pathogenesis is not understood thoroughly. To investigate the roles of MMP7 in colon cancer progression, we introduced an Mmp7 knockout mutation into the cis-Apc/Smad4 mutant mouse, a model of invasive colon cancer in which SMAD4-dependent TGF- $\beta$ family signaling is inactivated. We demonstrate here that lack of MMP7 reduces the number and size of tumors in the cis-Apc/Smad4 mice. On the other hand, MMP7-deficiency does not affect the depth of tumor invasion, number of stromal fibroblasts or levels of extracellular matrix components in the tumors. These results indicate that MMP7 is required for tumor formation, but not for the invasion or fibrosis of the colon cancer whose SMAD4-dependent TGF- $\beta$ family signaling is blocked.

Laboratory Investigation (2009) 89, 98-105; doi:10.1038/labinvest.2008.107; published online 10 November 2008

KEYWORDS: colon cancer; invasion; MMP7; SMAD4; TGF- $\beta$; tumor formation

Accumulating evidence suggest that most colorectal adenomas are initiated by the inactivation of $A P C$ gene, and progress into adenocarcinomas through additional genetic alterations in KRAS (for KRAS), TP53 (P53), SMAD4, TGFBR2 (TGF- $\beta$ type-II receptor), etc. ${ }^{1}$ In colorectal carcinomas, marked tumor invasion and expansion of the stroma are well-known features associated with metastasis and poor prognosis. $^{2-4}$

As a mouse model for colorectal adenocarcinomas, we previously constructed $c i s-A p c^{+/ \Delta 716} S m a d 4^{+/-}$compound mutant (hereafter cis-Apc/Smad4 mice) carrying a knockout allele of the Smad4 gene on the same chromatid as that of Apc. ${ }^{5}$ Using these mice, we demonstrated that loss of SMAD4-dependent TGF- $\beta$ family signaling converts intestinal adenomas to adenocarcinomas. Namely, the cis-Apcl Smad4 mice develop tumors that show marked invasion and stromal expansion, although the simple $A p c$ mutant $\left(A p c^{\Delta 716}\right)$ mice form only benign adenomas. ${ }^{5,6}$ To examine the mechanisms underlying such malignant changes in the cis-Apc/Smad4 tumors, we recently performed DNA microarray analyses and found that the adenocarcinomas expressed much higher levels of CC-chemokine ligand 9 (CCL9) than the $A p c^{\Delta 716}$ adenomas. Further studies revealed that CCL9 released from the cis-Apc/Smad4 tumor epithelia recruits immature myeloid cells (iMCs) that carry its CC-chemokine receptor (CCR1) and express matrix metalloproteinase (MMP) 2 and 9 to help tumor invasion. ${ }^{7}$

Although it is the tumor stromal cells that produce most MMPs whose levels are increased in the colon cancer (eg MMP2 and MMP9), the tumor epithelial cells can express another MMP, MMP7. ${ }^{8}$ Interestingly, our microarray data ${ }^{7}$ showed increased expression of MMP7 in the cis-Apc/Smad4 adenocarcinomas compared with the $A p c^{\Delta 716}$ adenomas. In human colorectal cancer, it has been reported that the level of MMP7 correlates with advanced Dukes stages, poor outcome, invasion and metastasis to the liver. ${ }^{9-11}$ Several studies have shown that overexpression of MMP7 increases the invasive ability and metastasis to the liver in some colon cancer cell lines, ${ }^{9,12,13}$ although another study suggests that MMP7 has little effect on the invasive potential or metastasis capacity of colon cancer cells. ${ }^{14}$ Interestingly, a recent report suggests that MMP7 can contribute to expansion of the tumor

\footnotetext{
${ }^{1}$ Department of Pharmacology, Graduate School of Medicine, Kyoto University, Kyoto, Japan and ²Devision of Genetics, Cancer Research Institute, Kanazawa University, Kanazawa, Japan

Correspondence: Professor MM Taketo, MD, PhD, Department of Pharmacology, Graduate School of Medicine, Kyoto University, Yoshida-Konoe-cho, Sakyo, Kyoto 6068501, Japan.

E-mail: taketo@mfour.med.kyoto-u.ac.jp

${ }^{3}$ These authors contributed equally to this work.

Received 9 July 2008; revised 5 September 2008; accepted 16 September 2008
} 
stroma, because recombinant MMP7 stimulates proliferation and migration of human colonic fibroblasts. ${ }^{15}$ As these studies relied on cell lines, however, it remains to be determined what actual roles MMP7 plays in colon cancer development in vivo.

To address this issue, we crossed the cis-Apc/Smad4 mice with the $M m p 7^{-1-}$ and generated triple mutant mice, cis-Apc/Smad4 $\mathrm{Mmp}^{-1-}$. Here, we report that lack of MMP7 in the cis-Apc/Smad4 mice reduces the number and size of tumors, but does not affect the depth of invasion, number of stromal fibroblasts or expression of extracellular matrix components.

\section{MATERIALS AND METHODS Knockout Mice}

The knockout alleles of the Apc, Smad4 and Mmp7 genes have been described previously. ${ }^{5,16,17}$ The cis-Apc/Smad4 male mice were crossed twice with the $M m p 7^{-1-}$ female mice (from Jackson Laboratory, Bar Harbor, ME, USA) to generate triple mutant $c i s-A p c / S m a d 4 M m p 7^{-1-}$ mice. All animal experiments have been conducted according to the protocol approved by the Animal Care and Use Committee of Kyoto University.

\section{RT-PCR}

The levels of mRNAs were determined by RT-PCR as described previously, ${ }^{7}$ using the following primers: $M m p 2$ (F: $5^{\prime}$-GCAAGTTCCCGTTCCGCTTCC- $3^{\prime}$ and R: $5^{\prime}$-CAGTA CCAGTGTCAGTATCAGC-3'), Mmp3 (F: $5^{\prime}$-TGGAGATGCT CACTTTGAC G- $3^{\prime}$ and R: $5^{\prime}$-GATGGAAGAGATGGCCAAA A-3'), Mmp7 (F: $5^{\prime}$-GCGGAGATGCTC ACTTTGAC- $3^{\prime}$ and R: 5'-GCATCTATCACAGCGTGTTC-3'), Mmp9 (F: 5'-TACA GGGCCCCTTCCTTACT-3' and R: 5'-ACTCCTTATCCACG CGAATG-3'), Mmp10 (F: $5^{\prime}$ - CAGGTGTGGTGTTCCTGA TG- $3^{\prime}$ and R: $5^{\prime}$ - TGGAAGTTAGCTGGGCTTGT- $3^{\prime}$ ), Mmp14

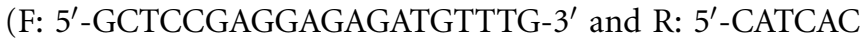
TGCCCATGAA TGAC-3'), Colla1 (F: 5'-CACCCTCAAGAG CCTGAGTC $-3^{\prime}$ and R: $5^{\prime}$-GGTGGAGG GAGTTTACACG A- $\left.3^{\prime}\right), \quad$ Colla2 (F: $5^{\prime}$-TGGATTGACCCTAACCAAGG- $3^{\prime}$ and R: $5^{\prime}$-CTTGCCCCATTCATTTGTCT-3') and Gapdh (F: $5^{\prime}$-CCTTCATTGACCTCAACTAC- $3^{\prime}$ and R: $5^{\prime}$-TGGGC C CTCAGATGCCTGCT-3').

\section{Western Blotting}

Dissected tumors were homogenized in lysis buffer containing $50 \mathrm{mM}$ Hepes, $150 \mathrm{mM} \mathrm{NaCl}, 20 \mathrm{mM} \mathrm{NaF}, 10 \mathrm{mM}$ sodium pyrophosphate, $2 \mathrm{mM}$ sodium vanadate, $5 \mathrm{mM}$ EDTA, 1\% NP-40 and a protease inhibitor cocktail (Roche, Indianapolis, IN, USA). The tissue lysates were separated in a sodium dodecyl sulfate-polyacrylamide gel $(15 \%)$ and transferred onto polyvinylidene difluoride membrane, using standard techniques. Specific signals were detected by rabbit polyclonal anti-MMP7 antibody (Calbiochem, San Diego, CA, USA) and mouse monoclonal anti $\beta$-actin antibody (Sigma, St Louis, MO, USA).

\section{Polyp Number Scoring}

The intestinal tissues were fixed with $4 \%$ paraformaldehyde and then transferred to PBS. The tumor diameters were measured under a dissection microscope, and the tumor numbers were scored for the respective size classes. Under a dissection microscope, we identified and scored the nascent polyps $(0.2-0.5 \mathrm{~mm}$ in diameter $)$ consisted of microadenomas. ${ }^{6}$

\section{Immunohistochemistry}

The intestinal tissues were fixed with $4 \%$ paraformaldehyde and embedded in paraffin wax for immunohistochemistry, or directly embedded in OCT compound (Sakura Finetek, Tokyo, Japan) for immunofluorescent staining. Sections were immunostained as described previously, ${ }^{7}$ using the following primary antibodies: rabbit polyclonal antibodies for MMP7 (Calbiochem), collagen type-I (LSL, Tokyo, Japan), fibronectin (Sigma), tenascin-C (Sigma) and pan-cytokeratin (Dako, Carpinteria, CA, USA), and rat monoclonal antibodies for CD31, CD34 and CD11b (BD Biosciences, San Jose, CA, USA). In the immunofluorescent staining, nuclei were detected by staining with DAPI (Calbiochem).

\section{Counting Fibroblasts}

Frozen sections of the intestinal polyps were stained with rat monoclonal antifibroblast antibody (AbD Serotec, Raleigh, NC, USA) as described above. The nuclei in the stained cells were counted under a fluorescent microscope $(\times 400)$. Nine randomly selected fields were scored per mouse. Results were presented as the mean number of cells per field obtained from three mice for each strain.

\section{Statistical Analysis}

The values were shown as mean \pm s.d., and analyzed by Student's $t$-test. The ' $P$ '-values smaller than 0.05 were considered significant.

\section{RESULTS \\ Expression of MMP7 is Increased in cis-Apc/Smad4 Intestinal Tumors}

To investigate the mechanism of tumor invasion and stromal expansion in the cis-Apc ${ }^{+/ \Delta 716} \mathrm{Smad4}^{+/-}$(cis-Apc/Smad4) adenocarcinomas, we recently performed DNA microarray analyses $^{7}$ and found higher expression of $M m p 7$ mRNA in the carcinomas than in the $A p c^{\Delta 716}$ adenomas. To confirm the microarray data, we performed RT-PCR and western blotting. As shown in Figure 1a, the Mmp7 mRNA level in the cis-Apc/Smad4 adenocarcinomas was three times higher than that in the $A p c^{\Delta 716}$ adenomas. Expression of the $M m p 7$ mRNA in the $A p c^{\Delta 716}$ adenomas was twice higher than in the normal intestinal tissues of the wild-type C57BL/6 mice, although to a less extent than in the cis-Apc/Smad4 adenocarcinomas (sixfold). We also found that expression of the MMP7 precursor protein was increased moderately in the cis-Apc/Smad4 adenocarcinomas compared with the $A p c^{\Delta 716}$ 
a
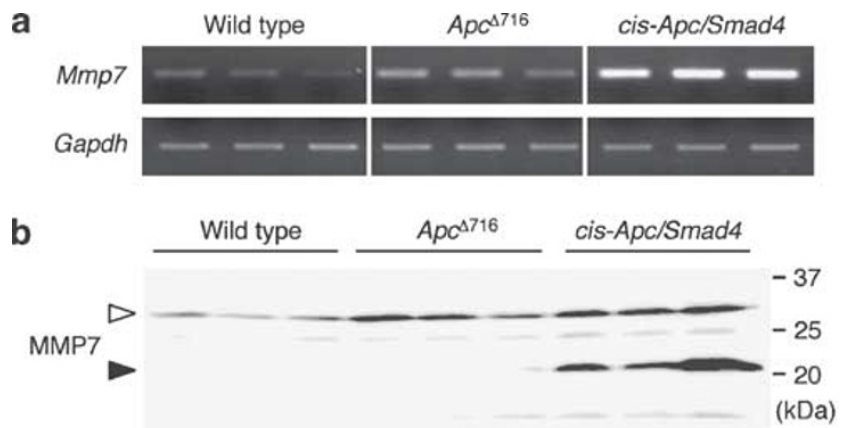

$\beta$-actin

C
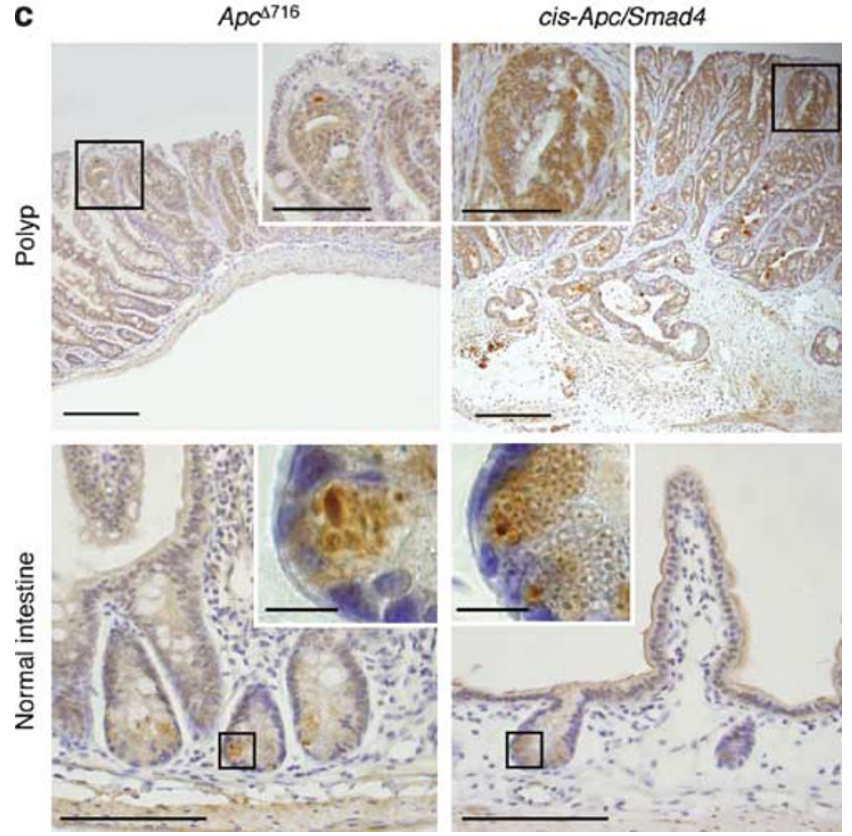

Figure 1 The levels of MMP7 in the cis-Apc/Smad4 polyps. (a) Expression of MMP7 mRNA determined by RT-PCR. Total RNA was prepared from the intestinal tissues of normal C57BL/6 mice (wild type) or polyps of $A p c^{\Delta 716}$ and cis-Apc/Smad4 mice. (b) Expression of MMP7 protein determined by western blotting. Open and closed arrowheads indicate pro- and activeMMP7, respectively. (c) Intestinal polyps (top) and normal tissues (bottom) of $A p c^{\Delta 716}$ (left) and cis-Apc/Smad4 (right) mice stained for MMP7. Insets show higher magnifications of the boxed areas. Scale bars show 200 and $100 \mu \mathrm{m}$ (inset) in top panels, and 200 and $10 \mu \mathrm{m}$ (inset) in bottom panels.

adenomas (1.3-fold) or normal tissues (1.9-fold; Figure 1b). Furthermore, the levels of the cleaved (active) form MMP7 were markedly elevated in the $c i s-A p c / S m a d 4$ adenocarcinomas (27 times higher than in the $A p c^{\Delta 716}$ adenomas). Immunostaining data showed that the MMP7 protein localized to the tumor epithelial cells in both $A p c^{\Delta 716}$ and cis-Apc/ Smad4 polyps (Figure 1c). Consistent with the western blotting data, the cis-Apc/Smad4 adenocarcinoma cells showed a stronger immunoreactivity to MMP7 than the $A p c^{\Delta 716}$ adenoma cells. As described previously, ${ }^{18}$ Paneth cells in the normal intestinal crypt expressed MMP7 in both $A p c^{\Delta 716}$ and $c i s-A p c / S m a d 4$ mice at similar levels (Figure 1c). These results indicate that expression of MMP7 is increased
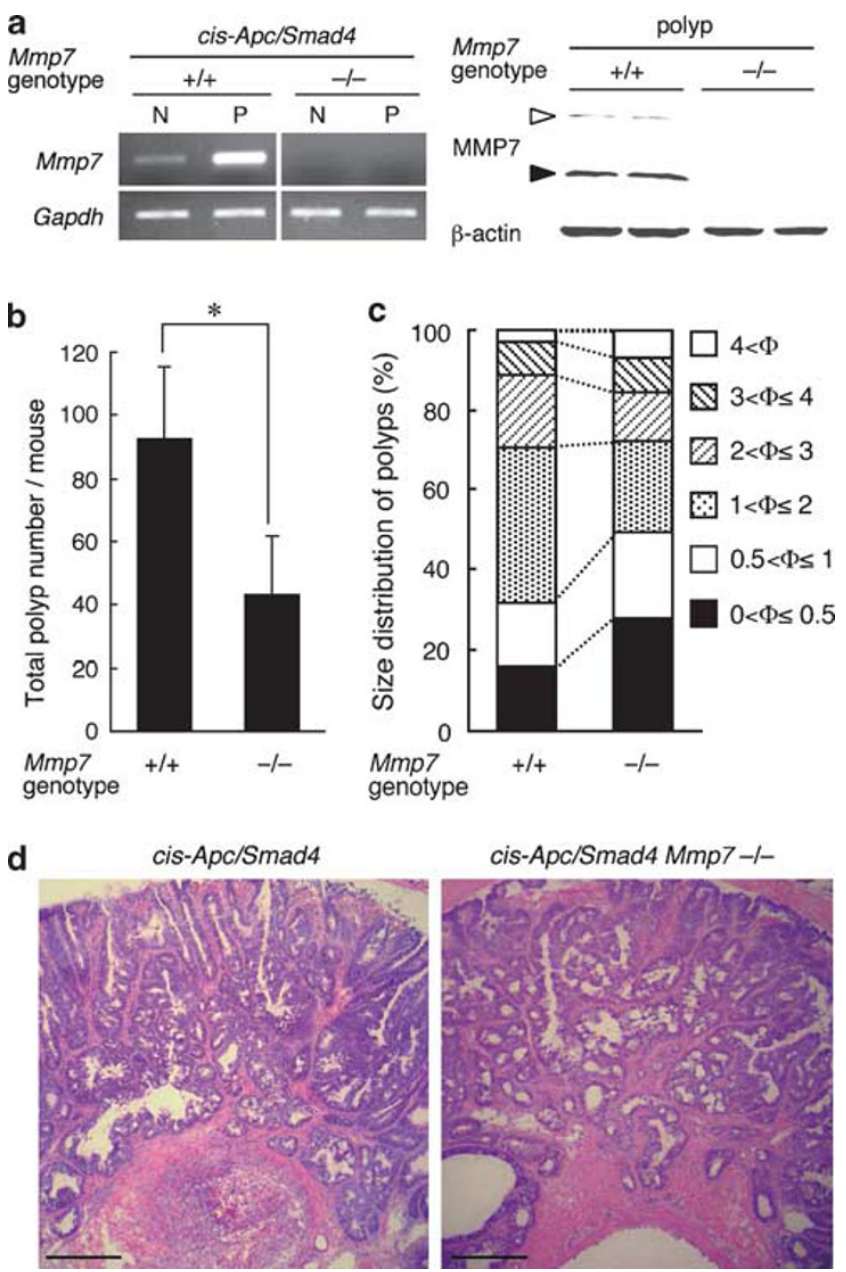

Figure 2 Effects of MMP7-deficiency on tumor formation in the cis-Apc/ Smad4 mice. (a) Expression of MMP7 mRNA (left) and protein (right) in the intestinal polyps (P) and normal tissues (N) from MMP7-intact $(+/+)$ and knockout (-/-) cis-Apc/Smad4 mice. (b) Total number of the intestinal polyps in the MMP7-intact $(+/+)$ and knockout $(-/-)$ cis-Apc/Smad4 mice. Results show a mean number of polyps/mouse \pm s.d. $(n=9)$. $P<0.001$. (c) Size distribution of the intestinal polyps. Polyps were classified according to their diameters in millimeters. Results are shown as percentages for the respective size classes $(n=9)$. $\Phi$ indicates polyp diameter (mm). (d) Intestinal tumors in the cis-Apc/Smad4 and cis-Apc/ Smad4 $\mathrm{Mmp}^{-1-}$ mice stained with H\&E. Scale bars show $200 \mu \mathrm{m}$.

during the progression of intestinal adenomas to adenocarcinomas, caused by impaired SMAD4 signaling in the cisApc/Smad4 mice.

\section{Loss of MMP7 Reduces Number and Size of Tumors in cis-Apc/Smad4 Mice}

To examine the role of the increased MMP7 expression in the development of SMAD4-deficient adenocarcinomas, we

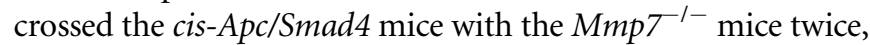
and generated triple mutant mice. As shown in Figure 2a, we found no expression of MMP7 mRNA or protein in the intestinal polyps of the cis-Apc/Smad4 $\mathrm{Mmp7}^{-1-}$ mice. Because some of these mice became moribund at the age of 


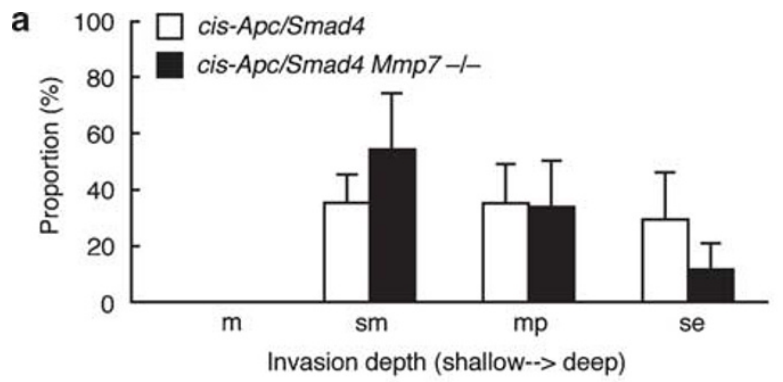

b

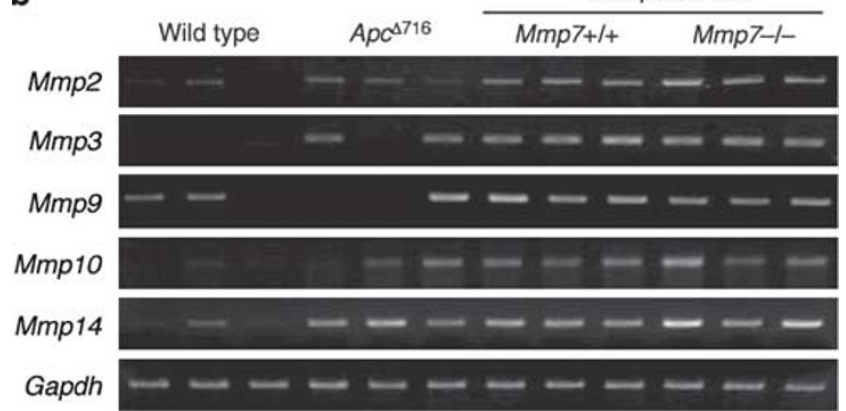

C cis-Apc/Smad4
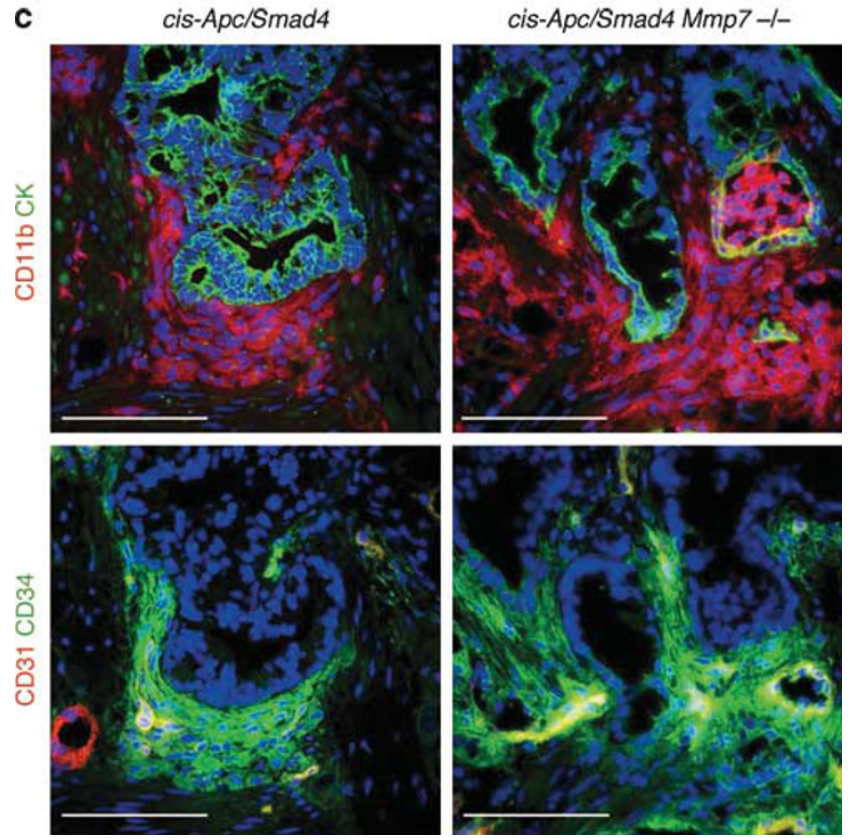

Figure 3 Intestinal tumor invasion in the cis-Apc/Smad4 $\mathrm{Mmp7}^{-1-}$ tumors. (a) The invasion depths of the polyps $(\Phi \geq 2 \mathrm{~mm})$ in the cis-Apc/Smad4 and cis-Apc/Smad4 $\mathrm{Mmp7}^{-1-}$ mice ( $n=50$ tumors from three mice per group). The polyps stained with $\mathrm{H}$ and $\mathrm{E}$ were classified according to their invasion depth, ie, mucosa $(\mathrm{m})$, submucosa $(\mathrm{sm})$, muscularis propria $(\mathrm{mp})$ and serosa (se). Results are presented as proportions among the polyps examined (mean \pm s.d. from 50 polyps in each strain). (b) Expression of MMP mRNAs determined by RT-PCR. Total RNA was prepared from the intestinal tissues of wild-type mice or $A p c^{\Delta 716}$, cis-Apc/Smad4 and cis-Apc/Smad4 $\mathrm{Mmp7}^{-1-}$ polyps. (c) Invasion front of the cis-Apc/Smad4 (left) and cis-Apc/Smad4 $\mathrm{Mmp7}^{-1-}$ (right) polyps. Serial sections were double stained for pancytokeratin (green) and CD11b (red) or CD34 (green) and CD31 (red), respectively. Scale bars show $100 \mu \mathrm{m}$.
14 weeks, we killed all animals at this age in this series of experiments.

As loss of MMP7 suppressed the tumorigenesis of the intestinal adenomas in $A p c^{\mathrm{Min}}$ mice, ${ }^{17}$ we first investigated whether the MMP7-deficiency affected the number or size of the adenocarcinomas in the cis-Apc/Smad4 mice. Consistent with our earlier report, ${ }^{5}$ the $c i s-A p c / S m a d 4$ mice developed $93 \pm 23$ intestinal polyps at the age of 14 weeks (Figure 2b). Compared with the cis-Apc/Smad4 mice, the cis-Apc/Smad4 $M m p 7^{-1-}$ mice had a $50 \%$ less number of tumors at the same age ( $43 \pm 19$ polyps, Figure $2 b$ ), suggesting that the lack of MMP7 suppressed tumor formation. Furthermore, we found smaller tumor size in the cis-Apc/Smad4 $\mathrm{Mmp7}^{-1-}$ mice (Figure 2c). Namely, the ratio of large polyps $(1.0-3.0 \mathrm{~mm}$ in diameter) in the cis-Apc/Smad4 $\mathrm{Mmp}^{-1-}$ mice (35\%) was significantly lower than that in the cis-Apc/Smad4 mice $(57 \%)$. On the other hand, the ratio of small polyps $(<1.0 \mathrm{~mm})$ was higher in the cis-Apc/Smad4 $\mathrm{Mmp}^{-1-}$ (49\%) than in the cis-Apc/Smad4 (32\%). It is worth noting that the ratio for very large polyps $(>3.0 \mathrm{~mm})$ was similar between the two strains (Figure 2c). As shown in Figure 2d, there was no obvious difference in the polyp morphology between the two types of mutants. These results suggest that MMP7 facilitates the expansion of most adenocarcinomas in the cis-Apc/Smad4 mice, although some tumors can expand without MMP7. Consistent with the data of $A p c^{\mathrm{Min}}$ mice, ${ }^{17}$ the absence of MMP7 reduced the number and size of tumors in $A p c^{\Delta 716}$ mice, as well (data not shown).

\section{Loss of Mmp7 does not Affect Invasion Depth of cis-Apc/ Smad4 Tumors}

We have demonstrated previously that the cis-Apc/Smad4 polyps show marked invasion, and that the invasion is helped by the iMCs that are recruited by CCL9 and express its receptor CCR1 as well as MMP2/9. ${ }^{7}$ Because increased expression of MMP7 can contribute to colorectal tumor invasion, ${ }^{9,12,13}$ we next studied the effect of MMP7-deficiency on the depth of tumor invasion in the cis-Apc/Smad4 tumors. In the cis-Apc/Smad4 mice, all tumors showed invasion into the submucosa, smooth muscle layer and serosa at the proportions of 35, 35 and 30\%, respectively (Figure 3a). Contrary to our anticipation, polyps in the cis-Apc/Smad4 $M m p 7^{-1-}$ mice showed marked invasions like those in the cis-Apc/Smad4 mice. Namely, 54 and $34 \%$ of the tumors invaded into the submucosa and smooth muscle layer, respectively (Figure 3a). Although the proportion of tumors that invaded to the serosa (12\%) was lower in the cis-Apcl Smad4 $\mathrm{Mmp}^{-1-}$ mutant, the difference was not significant statistically. Because it has been reported that the absence of MMP7 results in a compensatory increase in the level of MMP $2,{ }^{17}$ we examined the mRNA levels for MMPs involved in tumor invasion. In the cis-Apc/Smad4 $\mathrm{Mmp7}^{-1-}$ polyps, mRNA levels for Mmp2, Mmp3, Mmp9 and Mmp10 were much higher than those in the normal intestinal tissues or $A p c^{\Delta 716}$ polyps (Figure $3 \mathrm{~b}$ ). However, we could not find any 

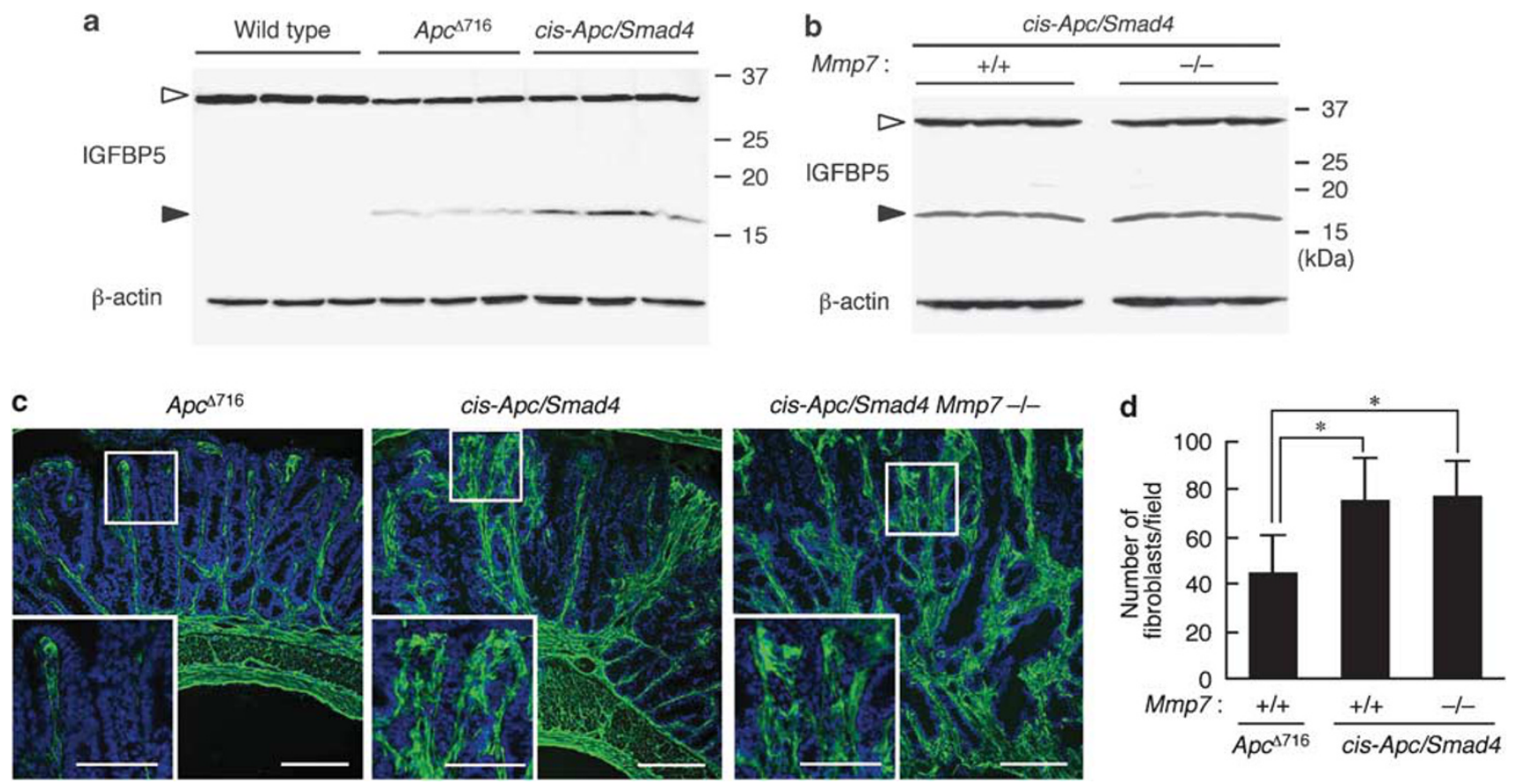

Figure 4 Effects of MMP7-deficiency on the number of stromal fibroblasts in the cis-Apc/Smad4 tumor. (a) Expression of IGFBP5 protein determined by western blotting. Protein samples were prepared from the intestinal tissues of wild-type mice or $A p c^{\Delta 716}$ and $c i s-A p c / S m a d 4$ polyps. Open and closed arrowheads indicate intact- and cleaved-IGFBP5, respectively. (b) Expression of IGFBP5 protein in the MMP7-intact $(+/+)$ and knockout $\left(^{-/-}\right)$cis-Apc/Smad4 polyps. (c) The intestinal polyps of the $A p c^{\Delta 716}$ (left), cis-Apc/Smad4 (middle) and cis-Apc/Smad4 $\mathrm{Mmp}^{-1-}$ (right) stained for fibroblast specific antigen. Insets show higher magnifications of the boxed areas. Scale bars show 200 and $100 \mu \mathrm{m}$ (inset). (d) The number of fibroblasts in the intestinal polyps. Genotypes of Mmp7 are shown as $+/+$ (wild type) or $-/-$ (knockout). Data are given as the mean numbers of fibroblasts per field $\pm s . d$. from nine fields per mouse $(n=3) . P<0.01$.

significant differences in the mRNA levels for these MMPs between the cis-Apc/Smad4 and cis-Apc/Smad4 $M m p 7^{-1-}$ polyps. Although the mRNA level for Mmp14 was slightly higher in the cis-Apc/Smad4 $M m p 7^{-1-}$ polyps than in the cisApc/Smad4 polyps (1.5-fold), the difference was not significant statistically. We next examined the recruitment of iMCs in the cis-Apc/Smad4 $\mathrm{Mmp}^{-1-}$ tumors. To this end, we performed immunostaining for CD34 and CD11b, markers for this class of cells. ${ }^{7}$ Consistent with our previous findings, we found accumulation of CD34- and CD11b-expressing iMCs at the invasion front of the cis-Apc/Smad4 polyps (Figure $3 \mathrm{~b}$ ). Similarly, the invading glands in the cis-Apc/ Smad4 $\mathrm{Mmp}^{-1-}$ adenocarcinomas were associated with iMCs (Figure 3b), suggesting that the cis-Apc/Smad4 tumor epithelial cells can attract the iMCs even in the absence of MMP7. These results indicate that increased expression of MMP7 in the tumor epithelium does not involve in the invasion of the adenocarcinomas in the cis-Apc/Smad4 mice.

\section{Lack of Mmp7 does not Affect Number of Fibroblasts in cis-Apc/Smad4 Tumor Stroma}

Expansion of the tumor stroma with fibroblasts and collagen fibers (fibrosis) is one of characteristic features in human colorectal carcinoma. ${ }^{2}$ Interestingly, MMP7 stimulates the proliferation of human colonic fibroblasts by cleaving insulin-like growth factor-binding protein 5 (IGFBP5) and subsequent release of insulin-like growth factor (IGF) ${ }^{15} \mathrm{We}$ therefore speculated that loss of MMP7 might prevent the cleavage of IGFBP5 and subsequent proliferation of fibroblasts in the cis-Apc/Smad4 adenocarcinomas. As shown in Figure $4 \mathrm{a}$, the cleaved form IGFBP5 was increased in the cisApc/Smad4 adenocarcinomas compared with that in the $A p c^{\Delta 716}$ adenomas. Furthermore, immunostaining showed a higher number of fibroblasts in the cis-Apc/Smad4 tumor stroma (75 \pm 18 cells per field) than in the $A p c^{\Delta 716}(44 \pm 16$; Figure $4 \mathrm{c}$ and $\mathrm{d}$ ). However, polyps in the triple mutant did not show much difference either in the extent of IGFBP5 cleavage or in the fibroblast numbers $(77 \pm 15)$ compared with those in the cis-Apc/Smad4 (Figure $4 \mathrm{~b}-\mathrm{d}$ ).

\section{Absence of Mmp7 does not Affect Accumulation of Collagen Type-I in cis-Apc/Smad4 Tumors}

In human colorectal carcinomas, fibronectin, tenascin and collagen type-I are major components of fibrotic foci, and are increased compared with normal tissues or adenomas. ${ }^{19-21}$ As shown in Figure 5a, we found more collagen type-I in the cis-Apc/Smad4 adenocarcinomas than in the $A p c^{\Delta 716}$ adenomas. Consistently, the mRNA levels for the $\alpha 1$ and $\alpha 2$ chains of collagen type-I (Colla1 and Colla2, respectively) were markedly higher in the cis-Apc/Smad4 adenocarcinomas (Figure 5b). Because it was reported that expression of MMP7 was increased in fibrosis of the liver and lungs, ${ }^{22,23}$ 
a

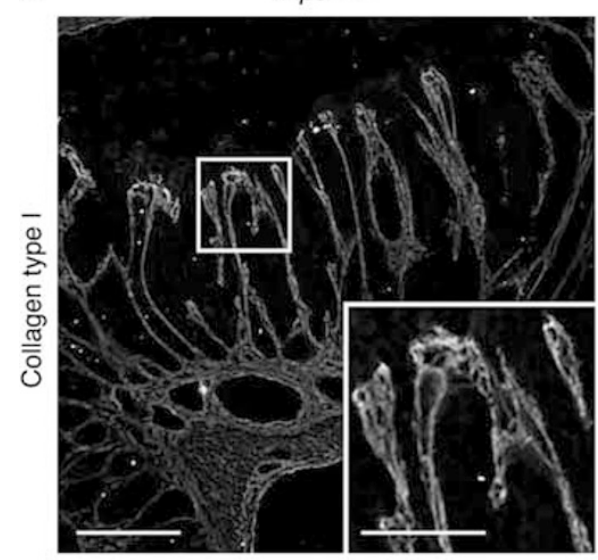

b

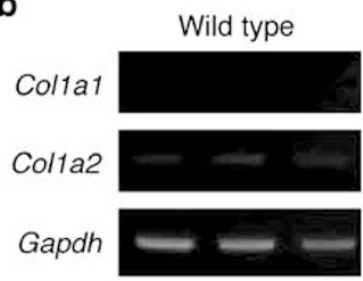

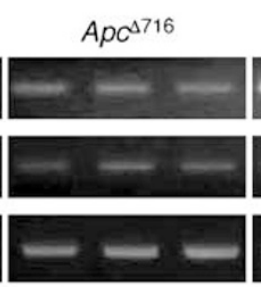

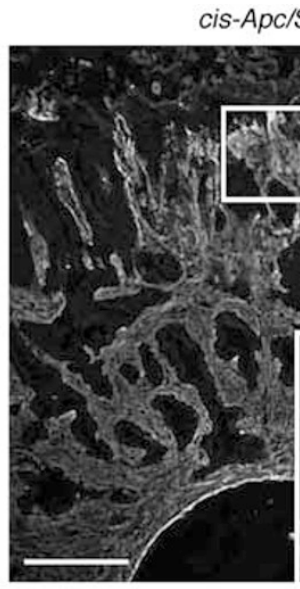

-Apc/Smad4
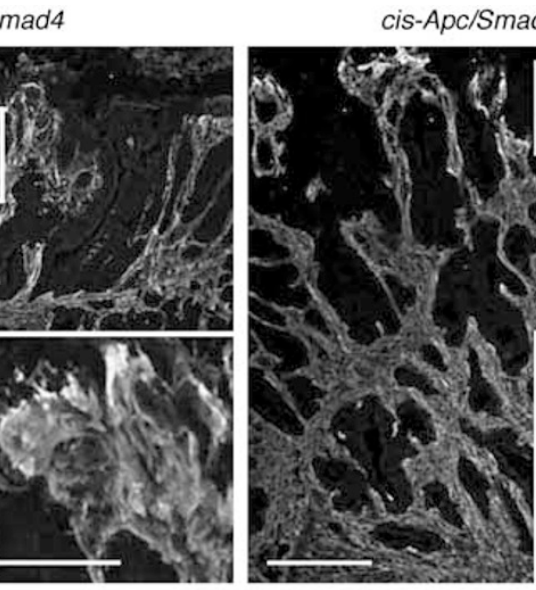

Mmp7-1-

cis-Apc/Smad4
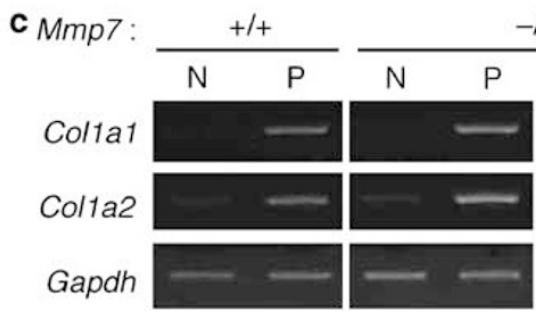

$-1-$

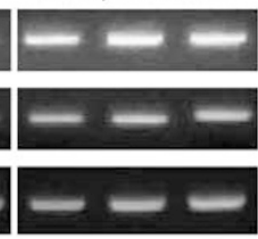

Gapdh

Figure 5 Expression of collagen type-I in the cis-Apc/Smad4 $\mathrm{Mmp}^{-1-}$ tumors. (a) The intestinal polyps of the $A p c^{\Delta 716}$ (left), cis-Apc/Smad4 (middle) and cisApc/Smad4 $\mathrm{Mmp}^{-1-}$ (right) stained for collagen type-I. Insets show higher magnifications of the boxed areas. Scale bars show 200 and $100 \mu \mathrm{m}$ (inset). (b) Expression of collagen type-I mRNA determined by RT-PCR. Col1a1 and Col1a2 show $\alpha 1$ and $\alpha 2$ chains of collagen type-I, respectively. Total RNA was prepared from the intestinal tissues of wild-type mice or $A p c^{\Delta 716}$ and $c i s-A p c / S m a d 4$ polyps. (c) Expression of collagen type-I mRNA in the intestinal polyps (P) and normal tissues $(\mathrm{N})$ from cis-Apc/Smad4 $(+/+)$ and cis-Apc/Smad4 $\mathrm{Mmp7}^{-1-}(-/-)$ mice.

and because loss of MMP7 reduced the severity of the bleomycin-induced pulmonary fibrosis, ${ }^{23}$ we next investigated the effect of MMP7-deficiency on the amount of collagen type-I in the $c i s-A p c / S m a d 4$ tumors. Contrary to our anticipation, the cis-Apc/Smad4 $M m p 7^{-1-}$ tumors showed essentially the same levels of collagen type-I protein and mRNA as the cis-Apc/Smad4 tumors (Figure 5a and c). Therefore, it appears unlikely that MMP7 contributes to the increased synthesis of collagen type-I in the cis-Apc/Smad4 adenocarcinomas.

Consistent with the clinical data, ${ }^{20}$ expression of fibronectin and tenascin was also increased in the cis-Apc/ Smad4 adenocarcinomas compared with the $A p c^{\Delta 716}$ adenomas (Figure 6). However, we found no distinct differences in their expression between the cis-Apc/Smad4 and cis-Apc/ Smad4 $\mathrm{Mmp}^{-1-}$ tumors. Although fibronectin is a known substrate of MMP7, ${ }^{24,25}$ these immunostaining data suggest that MMP7 is dispensable for its degradation in the intestinal tumors.

Taken together, the present results demonstrate that MMP7 promotes tumor formation, but it is not necessarily required for tumor invasion or fibrosis in the SMAD4-deficient intestinal adenocarcinomas.

\section{DISCUSSION}

We have demonstrated here that the tumor epithelial cells of the cis-Apc/Smad4 adenocarcinomas express higher levels of
MMP7 than those of the $A p c^{\Delta 716}$ adenomas. These cells lack the wild-type Smad4 alleles, and are devoid of the SMAD4dependent TGF- $\beta$ family signaling. ${ }^{5,7}$ Thus, our results with the mouse models suggest that inactivation of the TGF- $\beta$ family signaling stimulates expression of MMP7 during the course of progression from colon adenomas to carcinomas. Consistently, it has been reported that expression of MMP7 is increased in human colorectal carcinomas compared with adenomas. ${ }^{26,27}$

MMP7 has been implicated in benign colon adenomas. For example, lack of MMP7 reduces the number and size of the adenomas in $A p c^{\mathrm{Min}}$ mice. ${ }^{17}$ However, it remains to be investigated whether MMP7 inactivation also suppresses development of tumors carrying mutations in $A p c$ and an additional tumor suppressor (eg $p 53$, Smad4 or Tgfbr2). In this study, we have demonstrated that loss of MMP7 reduces both number and size of tumors in the cis-Apc/Smad4 mice, suggesting that inhibition of MMP7 can prevent tumor formation initiated by the $A p c$ inactivation in the presence of Smad4 mutation.

In human colon carcinomas, it has been reported that expression of MMP7 correlates with poor prognosis, advanced Dukes stage, liver metastasis and depth of invasion. ${ }^{9-11}$ Furthermore, cell line studies have shown that overexpression of MMP7 increases the invasiveness and metastatic potential of colon cancer cells, ${ }^{9,13}$ and that an antisense oligonucleotide against MMP7 suppresses liver metastasis. ${ }^{28}$ These results 

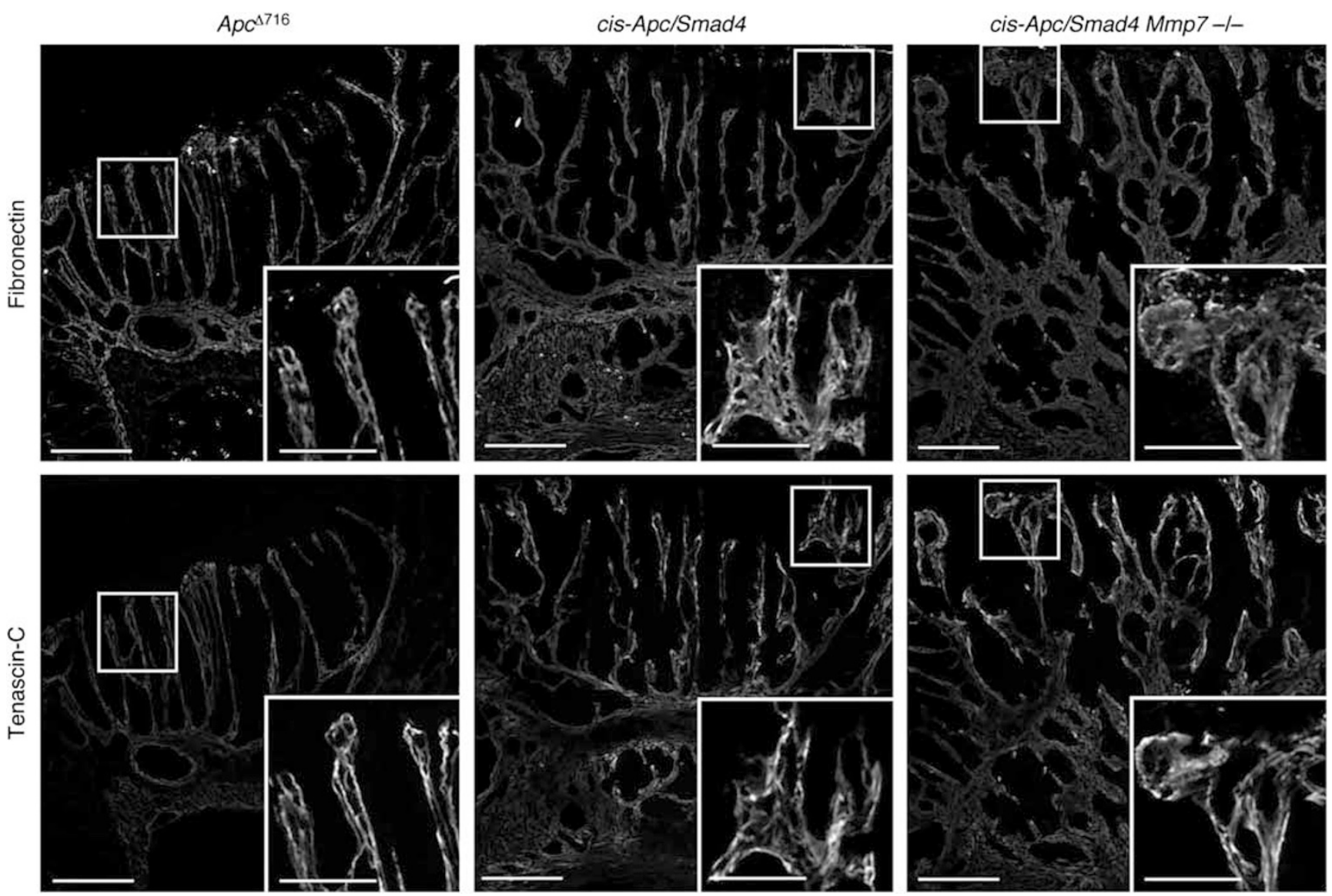

Figure 6 Expression of fibronectin and tenascin in the cis-Apc/Smad4 $M m p 7^{-1-}$ tumors. The intestinal polyps of the $A p c^{\Delta 716}$ (left), cis-Apc/Smad4 (middle) and cis-Apc/Smad4 $\mathrm{Mmp7}^{-1-}$ (right) stained for fibronectin (top) and tenascin (bottom). Insets show higher magnifications of the boxed areas. Scale bars show 200 and $100 \mu \mathrm{m}$ (inset).

suggest a significant role for MMP7 in tumor invasion and metastasis at late stages of colon cancer progression. On the other hand, we have shown here that lack of MMP7 does not suppress tumor invasion in the cis-Apc/Smad4 adenocarcinomas. It is worth noting that the cis-Apc/Smad4 adenocarcinomas do not carry mutations in the Ras $(\mathrm{K}-\mathrm{ras})^{5}$ or p53 (Trp53; data not shown) gene, suggesting that the tumors in the cis-Apc/Smad4 mice represent human colon carcinomas at relatively early stages. Thus, our results indicate that MMP7 is not required for invasion of such mouse tumors, and are consistent with the clinical data showing no significant correlation between MMP7 expression and depth of invasion in the early colorectal carcinomas. ${ }^{29}$ It is still conceivable, however, that MMP7 be involved in colon cancer progression such as metastasis beyond the stage examined here. Inhibitors against MMPs are in clinical development for various tumors and negative findings such as those reported in this study may provide an important framework of properly testing these drugs in the right group of patients.

In this study, we have shown that the cis-Apc/Smad4 adenocarcinomas show some fibrosis, ie, increased number of fibroblasts and higher levels of collagen type-I compared with the $A p c^{\Delta 716}$ adenomas. To investigate the mechanisms under- lying the tumor fibrosis, we analyzed mRNA samples from $A p c^{\Delta 716}$ and $c i s-A p c / S m a d 4$ polyps using DNA microarray. ${ }^{7}$ The results showed that almost all secreted factors, other than CCL9 ${ }^{7}$ and MMP7, were expressed at essentially the same levels between the two types of polyps. Consistent with such data, we found no differences between the $A p c^{\Delta 716}$ and cis-Apcl Smad4 polyps in the levels of mRNA for profibrotic growth factors such as TGF- $\beta$, IGF, connective tissue growth factor, or platelet derived growth factor (Supplementary Figure 1). It is unlikely that the increased levels of CCL9 in the cis-Apc/ Smad4 polyps stimulate the tumor fibrosis, because loss of its cognate receptor CCR1 does not affect the stromal expansion (data not shown). Accordingly, we have examined the possibility whether MMP7 can contribute to the tumor fibrosis through a posttranscriptional mechanism, eg, release or activation of profibrotic growth factors. Although lack of MMP7 reduces the experimental pulmonary fibrosis in a mouse model, ${ }^{23}$ the loss of MMP7 alone has not suppressed the fibrosis in the cis-Apc/Smad4 tumors. It is conceivable that other MMPs or proteinases compensate the defect in tumor fibrosis caused by the lack of MMP7. Further investigation will be required to elucidate the mechanism(s) underlying the stromal expansion in the cis-Apc/Smad4 polyps. 
Using the $c i s-A p c / S m a d 4$ mice, we have scrutinized here the role of MMP7 in the intestinal tumor progression in vivo. We have found that MMP7 promotes tumor expansion throughout development of the SMAD4-deficient intestinal adenocarcinomas, but it is not required for the invasion or fibrosis in these tumors. Although MMP7 is expressed essentially in the tumor epithelium, other invasion-associated MMPs such as MMP2 and MMP9 are primarily localized in the stromal cells in human colon cancer. ${ }^{8,30,31}$ In the cis-Apc/Smad4 mice, we have recently demonstrated that the MMP2/9-expressing stromal iMCs promote tumor invasion, without affecting the tumor size or multiplicity. ${ }^{7}$ Our findings, taken together, suggest separate roles for MMP7 and MMP2/9 in the development of some colon carcinomas. Namely, MMP7 supports the tumor formation, whereas MMP2/9 help tumor invasion of colon cancer whose SMAD4-dependent TGF- $\beta$ family signaling is inactivated. Furthermore, the present results support our novel therapeutic strategy against tumor invasion, which targets MMP-producing stromal iMCs with CCR1 inhibitors, rather than direct inhibition of MMPs produced by the tumor epithelial cells. ${ }^{32}$

Supplementary Information accompanies the paper on the Laboratory Investigation website (http://www.laboratoryinvestigation.org)

\section{ACKNOWLEDGEMENTS}

This work was supported by the Grant-in-Aid for Scientific Research from the Ministry of Education, Culture, Sports and Technology of Japan (M.M. Taketo). We thank Lynn M. Matrisian for making MMP7-knockout mice available at the Jackson Laboratory.

\section{DISCLOSURE/CONFLICT OF INTEREST}

The authors declared no conflict of interests.

1. Weinberg RA. The Biology of Cancer, Chapter 11, Garland Science: New York, 2007.

2. Nishimura $R$, Hasebe $T$, Tsubono $Y$, et al. The fibrotic focus in advanced colorectal carcinoma: a hitherto unrecognized histological predictor for liver metastasis. Virchows Arch 1998;433: 517-522.

3. Sis B, Sarioglu S, Sokmen S, et al. Desmoplasia measured by computer assisted image analysis: an independent prognostic marker in colorectal carcinoma. J Clin Pathol 2005;58:32-38.

4. Weinberg RA. The Biology of Cancer, Chapter 14 Garland Science: New York, 2007.

5. Takaku K, Oshima M, Miyoshi $\mathrm{H}$, et al. Intestinal tumorigenesis in compound mutant mice of both Dpc4 (Smad4) and Apc genes. Cell 1998;92:645-656.

6. Oshima $\mathrm{H}$, Oshima M, Kobayashi M, et al. Morphological and molecular processes of polyp formation in $A p c^{\Delta 716}$ knockout mice. Cancer Res 1997:57:1644-1649.

7. Kitamura $\mathrm{T}$, Kometani $\mathrm{K}$, Hashida $\mathrm{H}$, et al. SMAD4-deficient intestinal tumors recruit CCR $1^{+}$myeloid cells that promote invasion. Nat Genet 2007;39:467-475.

8. Wagenaar-Miller RA, Gorden L, Matrisian LM. Matrix metalloproteinases in colorectal cancer: is it worth talking about? Cancer Metastasis Rev 2004:23:119-213.

9. Adachi $\mathrm{Y}$, Yamamoto $\mathrm{H}$, Itoh $\mathrm{F}$, et al. Contribution of matrilysin (MMP-7) to the metastatic pathway of human colorectal cancers. Gut 1999:45:252-258
10. Adachi $\mathrm{Y}$, Yamamoto $\mathrm{H}$, Itoh $\mathrm{F}$, et al. Clinicopathologic and prognostic significance of matrilysin expression at the invasive front in human colorectal cancers. Int J Cancer 2001;95:290-294.

11. Zeng Z-S, Shu W-P, Cohen AM, et al. Matrix metalloproteinase-7 expression in colorectal cancer liver metastases: evidence for involvement of MMP-7 activation in human cancer metastases. Clin Cancer Res 2002;8:144-148.

12. Kioi M, Yamamoto K, Higashi S, et al. Matrilysin (MMP-7) induces homotypic adhesion of human colon cancer cells and enhances their metastatic potential in nude mouse model. Oncogene 2003;22:8662-8670.

13. Yamamoto $\mathrm{H}$, Itoh $\mathrm{F}$, Hinoda $\mathrm{Y}$, et al. Suppression of matrilysin inhibits colon cancer cell invasion in vitro. Int J Cancer 1995;61:218-222.

14. Witty JP, McDonnell S, Newell KJ, et al. Modulation of matrilysin levels in colon carcinoma cell lines affects tumorigenicity in vivo. Cancer Res 1994;54:4805-4812.

15. Hemers E, Duval C, McCaig C, et al. Insulin-like growth factor binding protein-5 is a target of matrix metalloproteinase-7: implications for epithelial-mesenchymal signaling. Cancer Res 2005;65:7363-7369.

16. Oshima M, Oshima H, Kitagawa K, et al. Loss of Apc heterozygosity and abnormal tissue building in nascent intestinal polyps in mice carrying a truncated Apc gene. Proc Natl Acad Sci USA 1995;92: 4482-4486.

17. Wilson $\mathrm{CL}$, Heppner $\mathrm{KJ}$, Labosky PA, et al. Intestinal tumorigenesis is suppressed in mice lacking the metalloproteinase matrilysin. Proc Natl Acad Sci USA 1997;94:1402-1407.

18. Wilson CL, Ouellette AJ, Satchell DP, et al. Regulation of intestinal $\alpha$ defensin activation by the metalloproteinase matrilysin in innate host defense. Science 1999;286:113-117.

19. Bode MK, Karttunen TJ, Mäkelä J, et al. Type I and III collagens in human colon cancer and diverticulosis. Scand J Gastroenterol 2000;35:747-752.

20. Hauptmann S, Zardi L, Siri A, et al. Extracellular matrix proteins in colorectal carcinomas. Lab Invest 1995;73:172-182.

21. Hewitt RE, Powe DG, Carter I, et al. Desmoplasia and its relevance to colorectal tumor invasion. Int J Cancer 1993;53:62-69.

22. Huang C-C, Chuang J-H, Chou M-H, et al. Matrilysin (MMP-7) is a major matrix metalloproteinase upregulated in biliary atresia-associated liver fibrosis. Mod Pathol 2005;18:941-950.

23. Zuo F, Kaminski N, Eugui E, et al. Gene expression analysis reveals matrilysin as a key regulator of pulmonary fibrosis in mice and humans. Proc Natl Acad Sci USA 2002;99:6292-6297.

24. Miyazaki K, Hattori $Y$, Umenishi $F$, et al. Purification and characterization of extracellular matrix-degrading metalloproteinase, Matrin (Pump-1), secreted from human rectal carcinoma cell line. Cancer Res 1990;50:7758-7764.

25. von Bredow DC, Nagle RB, Bowden GT, et al. Degradation of fibronectin fibrils by matrilysin and characterization of the degradation products. Exp Cell Res 1995;221:83-91.

26. Ishikawa T, Ichikawa $Y$, Mitsuhashi M, et al. Matrilysin is associated with progression of colorectal tumor. Cancer Lett 1996;107:5-10.

27. Newell KJ, Witty JP, Rodgers WH, et al. Expression and localization of matrix-degrading metalloproteinases during colorectal tumorigenesis. Mol Carcinog 1994;10:199-204.

28. Hasegawa S, Koshikawa N, Momiyama N, et al. Matrilysin-specific antisense oligonucleotide inhibits liver metastasis of human colon cancer cells in a nude mouse model. Int J Cancer 1998;76:812-816.

29. Masaki T, Matsuoka H, Sugiyama M, et al. Matrilysin (MMP-7) as a significant determinant of malignant potential of early invasive colorectal carcinomas. Br J Cancer 2001;84:1317-1321.

30. Collins HM, Morris TM, Watson SA. Spectrum of matrix metalloproteinase expression in primary and metastatic colon cancer: relationship to the tissue inhibitors of metalloproteinases and membrane type-1-matrix metalloproteinase. $\mathrm{Br} \mathrm{J}$ Cancer 2001;84:1664-1670.

31. Nielsen BS, Timshel S, Kjeldsen L, et al. 92 kDa type IV collagenase (MMP-9) is expressed in neutrophils and macrophages but not in malignant epithelial cells in human colon cancer. Int $J$ Cancer 1996;65:57-62.

32. Kitamura T, Taketo MM. Keeping out the bud guys: gateway to cellular target therapy. Cancer Res 2007;67:10099-10102. 